\title{
Constrained Simulations of the Local Universe
}

\author{
G. Yepes ${ }^{1}$, L. Martinez-Vaquero ${ }^{1}$, Y. Hoffman ${ }^{2}$, \\ S. Gottlöber ${ }^{3}$, A. Klypin ${ }^{4}$ \\ ${ }^{1}$ Universidad Autónoma de Madrid \\ ${ }^{2}$ Hebrew University Jerusalem \\ ${ }^{3}$ Astrophysikalisches Institut Potsdam \\ ${ }^{4}$ New Mexico State University
}

\begin{abstract}
We have studied the formation of the Local Universe using constrained cosmological simulations. To this end we use the observed local mass and velocity distributions to generate the initial density field. A variety of N-body simulations with different box sizes and resolutions have been done so far. The largest structures, such as Coma, Virgo and the Local Supercluster are reproduced with high accuracy. We have also been able to obtain a representation of the Local Group with unprecedented accuracy and resolution. From these simulations we are studying in detail the dynamics of the dark matter in our neighborhood. The next step will be to include baryons and study in detail the galaxy formation in our local universe.
\end{abstract}

\section{Introduction}

Cosmological simulations are the best tool to study the formation of galaxies and other structures in the Universe from cosmological initial conditions. Due to the stochastic nature of the gravitational growth of density fluctuations, we cannot dream to reproduce the exact initial conditons that gave rise to the structures that we see close to where we are in the Universe, namely the Local Group (LG) and the Local Supercluster (LSC). There is, nevertheless a technique to impose observational constrains on the density and velocity fields in a randomly generated realization of a cosmological gaussian random fluctation field Hoffman \& Ribak (1991). To this end, the smaller Fourier modes of the random power spectrum of density fluctuations are substituted by the linear extrapolated, smoothed density field obtained from the observational constrains, in order to recover our real environment. The observational data we use come from MARK III (Willick et al. 1997), SBF (Tonry et al. 2001), nearby X-ray clusters of galaxies (Reiprich \& Böhringer 2002) and the Karachentsev (2005) catalogues.

\section{Simulations}

Different N-body simulations, with different resolutions ( $256^{3}$ to $4096^{3}$ particles), box sizes (64 to $160 h^{-1} \mathrm{Mpc}$ ), codes (GADGET and ART) and goals, have been performed up to now. Using the low resolution N-body simulations with constrained and unconstrained initial conditions we have studied the correlations between the kinematics and dynamics of the LG candidates found by Martinez-Vaquero, Yepes and Hoffman (2007). We concluded that the lack of correlation between peculiar velocities and Newtonian accelerations due to galaxies within the local volume ( $7 \mathrm{Mpc}$ sphere around us) is a natural prediction of the Cold Dark Matter dominated models and that there is a strong bias between the local Newtonian forces and the "real" ones due to the tidal fields and the diffuse matter located in the tiny filaments linking the galactic halos. 


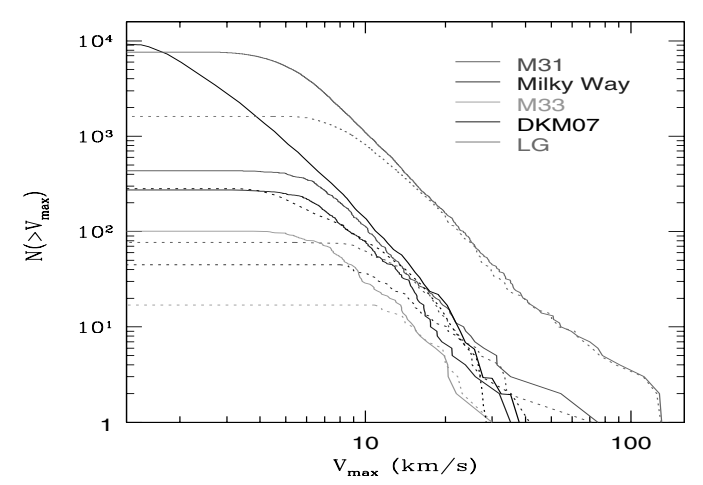

Figure 1. The cumulative maximum circular velocity function of subhalos within each of the main halos of the $4096^{3}$ LG simulation and the whole LG system. The dashed lines correspond to results from our LG simulation with 8 times bigger mass particles (2048 ${ }^{3}$ effective particles). The black solid curve correspond to the "Via Lactea" N-body simulation.

Our most accurate realization consists of a resimulated region of the order of $2 \mathrm{Mpc}$ around the best Local Group candidate found in a $64 h^{-1}$ Mpc box which consists of 3 main halos which have a very good resemblance of the Milky Way-Andromeda-M33 system. The simulation consists of more than 60 million particles in the resimulated area with a mass resolution of $250,000 M_{\odot}$ per particle. This means that each of the main components of the LG is resolved with more than 2 million particles. In Fig 1 we represent the substructure mass functions and compare them with the "Via Lactea" simulation results (Diemand, Kuhlen \& Madau, 2007) which has a mass resolution somewhat higher than our simulation. The cumulative function for the total LG system is also shown for comparison.

\section{References}

Diemand, J., Kuhlen, M \& Madau, P 2007 ApJ, 657, 262

Hoffman, Y., \& Ribak, E. 1991, ApJ, 380, 5

Karachentsev, I. D. 2005, AJ, 129, 178

Martinez-Vaquero, L. A., Yepes, G., \& Hoffman, Y., 2007, MNRAS, 378, 1601

Reiprich, T. H., \& Böhringer, H. 2002, ApJ, 567, 716

Tonry, J. L., et al., 2001, ApJ, 546, 681

Willick, J. A., et al., 1997], ApJ, 109, 333 